\title{
Adaptive bandwidth selection in the long run covariance estimator of functional time series
}

\author{
Lajos Horváth, Gregory Rice, Stephen Whipple \\ Department of Mathematics, University of Utah, Salt Lake City, UT 84112-0090 USA
}

\begin{abstract}
In the analysis of functional time series an object which has seen increased use is the long run covariance function. It arises in several situations, including inference and dimension reduction techniques for high dimensional data, and new applications are being developed routinely. Given its relationship to the spectral density of finite dimensional time series, the long run covariance is naturally estimated using a kernel based estimator. Infinite order "flattop" kernels remain a popular choice for such estimators due to their well documented bias reduction properties, however it has been shown that the choice of the bandwidth or smoothing parameter can greatly affect finite sample performance. An adaptive bandwidth selection procedure for flattop kernel estimators of the long run covariance of functional time series is proposed. This method is extensively investigated using a simulation study which both gives an assessment of the accuracy of kernel based estimators for the long run covariance function and provides a guide to practitioners on bandwidth selection in the context of functional data.
\end{abstract}

Keywords: functional data, long run covariance, mean squared error, optimal bandwidth

\section{Introduction}

A common way of obtaining functional data is to break long, continuous records into a sample of shorter segments which may be used to construct curves. For example, tick data measuring the price of an asset obtained

\footnotetext{
Research supported by NSF grant DMS 1305858

Email address: rice@math.utah.edu (Gregory Rice)
} 
over several years, which in principle may contain millions of data points, may be used to construct smaller samples of daily or weekly curves. Over the last decade functional time series analysis has grown steadily due to the prevalence of these types of data; we refer to [8] and [11] for a review of the subject.

Suppose

$X_{i}(t), 1 \leq i \leq n$ and $t \in[0,1]$ are observations from

a stationary ergodic functional time series with $E\left\|X_{0}\right\|^{2}<\infty$,

where $\|\cdot\|$ denotes the standard norm in $L^{2}$. An object which arises frequently in this context is the long run covariance function

$$
C(t, s)=\sum_{i=-\infty}^{\infty} \operatorname{Cov}\left(X_{0}(t), X_{i}(s)\right), 0 \leq t, s \leq 1 .
$$

$C$ may be viewed as an extension of the spectral density function evaluated at zero for univariate and multivariate time series, and its usefulness in the analysis of functional time series is similarly motivated. For example, under some regularity conditions $\sqrt{n} \bar{X}(t)$ is asymptotically Gaussian with covariance function $C$, where

$$
\bar{X}(t)=\frac{1}{n} \sum_{i=1}^{n} X_{i}(t),
$$

and hence the distribution of functionals of $\bar{X}$ can be approximated using an approximation of $C$, see [13] and [10]. Also, the principle components computed as the eigenfunctions of the Hilbert-Schmidt integral operator

$$
c(f)(t)=\int_{0}^{1} C(t, s) f(s) d s
$$

may be used to give asymptotically optimal finite dimensional representations of dependent functional data, see [6]. Given its representation as an infinite 
sum, $C$ is naturally estimated with a kernel estimator of the form

$$
\hat{C}_{n, h}(t, s)=\sum_{i=-(n-1)}^{n-1} K\left(\frac{i}{h}\right) \hat{\gamma}_{i}(t, s),
$$

where

$$
\hat{\gamma}_{i}(t, s)= \begin{cases}\frac{1}{n} \sum_{j=1}^{n-i}\left(X_{j}(t)-\bar{X}(t)\right)\left(X_{j+i}(s)-\bar{X}(s)\right), & i \geq 0 \\ \frac{1}{n} \sum_{j=1-i}^{n}\left(X_{j}(t)-\bar{X}(t)\right)\left(X_{j+i}(s)-\bar{X}(s)\right), & i<0 .\end{cases}
$$

We use the standard convention that $\hat{\gamma}_{i}(t, s)=0$ when $i \geq n$. It was shown in [9] that if

$$
\begin{aligned}
& K(0)=1, K(u)=K(-u), K(u)=0 \text { if }|u|>c \text { for some } c>0, \\
& K \text { is continuous on }[-c, c]
\end{aligned}
$$

and

$$
h=h(n) \rightarrow \infty, \quad h=o(n), \text { as } n \rightarrow \infty,
$$

then

$$
\left\|\hat{C}_{n, h}-C\right\|=o_{P}(1)
$$

as long as $\left\{X_{i}(t)\right\}_{i=-\infty}^{\infty}$ is a weakly dependent Bernoulli shift (cf. (2.5)-(2.7)).

Although the $L^{2}$ consistency of $\hat{C}_{n, h}$ holds under these standard conditions on the kernel $K$ and the bandwidth parameter $h$, their choice can greatly affect the estimators performance in finite samples. Classically, finite order kernels such as the Bartlett and Parzen kernels were used, see [20]. More recently though infinite order "flat-top" kernels of the form

$$
K_{f}(t ; x)=\left\{\begin{array}{lr}
1, & 0 \leq|t|<x \\
(x-1)^{-1}(|t|-1), & x \leq|t|<1 \\
0, & |t| \geq 1
\end{array}\right.
$$


which are equal to one in a neighborhood of the origin and then decay linearly to zero, were advocated for by [17], [18] where it is shown that they give reduced bias and faster rates of convergence when compared to kernels of finite order.

An important consideration though, regardless of the kernel choice, is the selection of the bandwidth parameter $h$. At present there is no available guidance regarding the choice of the bandwidth parameter for kernel based estimation with functional data.

One popular technique for such problems is cross validation, which has been used with success in scalar spectral density estimation (cf. [3]). Such methods are difficult to extend to the functional setting however since the already time consuming calculations involved in applying cross validation with scalar data become incalculable with densely observed curves. A separate approach which is more amenable with functional data is the use of plug-in or adaptive bandwidths which aim to minimize the mean squared error using an estimated bandwidth. Among the contributions in this direction are [1], [2], and [5] who showed that the asymptotically optimal bandwidth for spectral density estimation with scalar $\operatorname{ARMA}(p, q)$ data using finite order kernels is of the form $c_{d} n^{1 / r}$, where $c_{d}$ increases with the strength of dependence of the sequence. Their results are established by comparing the estimators asymptotic bias, which can be computed with standard arguments, to the asymptotic variance for which formulae have been derived in the scalar case, see [19]. This theory and subsequent simulation studies all indicate that the bandwidth should increase with the level of dependence in the data. In case of kernels of infinite order, [16] developed an adaptive bandwidth selection procedure which utilizes the correlogram.

The goal of this paper is to develop and numerically investigate an adaptive bandwidth selection procedure for the flat-top kernel estimator of the long run covariance of functional time series. Our procedure is motivated by the exact asymptotic order of the integrated variance of the estimator $\hat{C}_{n, h}(t, s)$ which we establish in Section 2 for a broad class of functional time series. This result is of interest in its own right since it may be used subsequently to derive optimal plug-in bandwidths for arbitrary kernels satisfying (1.4). In Section 3 we develop a bandwidth selection procedure for the flattop kernel (1.7). A thorough simulation study is given in Section 4 which compares our procedure to several fixed bandwidth choices available in the literature. In Subsection 4.3 we illustrate an application of the methodology developed in the paper to densely recorded stock price data for Citigroup. 
The paper concludes with some technical derivations which are contained in Section 5.

\section{Asymptotic integrated variance of the long run covariance es- timator}

The primary goal of bandwidth selection for scalar spectral density estimation has been to minimize the mean squared error of the kernel estimator. In case of square integrable functional data, error is usually measured using the standard $L^{2}$ norm, and hence we take the goal of bandwidth selection in this setting to be to minimize the integrated mean squared error $E\left\|\hat{C}_{n, h}-C\right\|^{2}$. Recognizably, the integrated mean squared error can be written as the sum of a variance term and a bias term

$$
E\left\|\hat{C}_{n, h}-C\right\|^{2}=\iint \operatorname{Var}\left(\hat{C}_{n, h}(t, s)\right) d t d s+\left\|E \hat{C}_{n, h}-C\right\|^{2},
$$

where $\int$ denotes $\int_{0}^{1}$. As with scalar data, one can show that the variance term is increasing with $h$ while the bias is decreasing with $h$, and thus the optimal bandwidth choice serves to balance the two quantities to give the fastest possible rate of convergence to zero. The bias is typically the simpler of the two terms to handle since $E \hat{C}_{n, h}-C$ can be computed explicitly in many cases. On the other hand, the variance in general cannot be computed explicitly and hence in most calculations it is exchanged with its asymptotic rate. In case of finite dimensional data the asymptotic rate of the variance of the kernel spectral density estimator is established under cumulant conditions which we now generalize to the functional setup. Since $\hat{C}_{n, h}$ does not depend on $E X_{0}(t)$, we can assume without loss of generality that

$$
E X_{0}(t)=0
$$

Let $a_{\ell}(t, s)=E X_{0}(t) X_{\ell}(s)$. We define the fourth order cumulant function as

$$
\begin{aligned}
\Psi_{\ell, r, p}(t, s)=E\left[X_{0}(t)\right. & \left.X_{\ell}(s) X_{r}(t) X_{p}(s)\right] \\
& -a_{\ell}(t, s) a_{p-r}(t, s)-a_{r}(t, t) a_{p-\ell}(s, s)-a_{p}(t, s) a_{r-\ell}(t, s) .
\end{aligned}
$$

Note that if the functional observations are simply scalars, i.e. $X_{i}(t)=X_{i}$, then $\Psi_{\ell, r, p}$ reduces to the scalar fourth order cumulant, see [19]. Under 
summability conditions of the integrals of the $a_{j}^{\prime} s$ and the fourth order cumulants asymptotics for $\iint \operatorname{Var}\left(C_{n, h}(t, s)\right) d t d s$ can be established.

Theorem 2.1. If (1.1), (1.4), (1.5), (2.1) hold, $E\left\|X_{0}\right\|^{4}<\infty$,

$$
\sum_{\ell=1}^{\infty}\left\|a_{\ell}\right\|<\infty
$$

and

$$
\frac{1}{h} \sum_{g, \ell=-h}^{h} \sum_{r=-(n-1)}^{n-1}\left|\iint \Psi_{\ell, r, r+g}(t, s) d t d s\right| \rightarrow 0
$$

as $n \rightarrow \infty$, then

$$
\begin{aligned}
& \lim _{n \rightarrow \infty} \frac{n}{h} \iint \operatorname{Var}\left(\hat{C}_{n, h}(t, s)\right) d t d s \\
&=\left(\|C(t, s)\|^{2}+\left(\int C(t, t) d t\right)^{2}\right) \int_{-c}^{c} K^{2}(t) d t
\end{aligned}
$$

In similar results with finite dimensional data (2.3) is replaced by a condition on the tri-infinite summability of the fourth order cummulants of the form

$$
\sum_{i, j, k=-\infty}^{\infty}\left|\iint \Psi_{i, j, k}(t, s) d t d s\right|<\infty
$$

from which (2.3) would follow by (1.5). Such a condition is exceedingly difficult to check, even for univariate data. However, (2.2) and (2.3) are satisfied for a class of weakly dependent random functions known as $L^{4}$ $\mathrm{m}$-approximable Bernoulli shifts. This class includes the functional ARMA, $\mathrm{ARCH}$, and GARCH processes under mild conditions. We say that $\mathbf{X}=$ $\left\{X_{j}(t)\right\}_{j=-\infty}^{\infty}$ is an $L^{4}-\mathrm{m}$-approximable Bernoulli shift (in $\left\{\epsilon_{j}(t),-\infty<j<\right.$ $\infty\})$ with rate $\alpha$ if

$X_{i}=g\left(\epsilon_{i}, \epsilon_{i-1}, \ldots\right)$ for some nonrandom measurable function

$g: S^{\infty} \mapsto L^{2}$ and i.i.d. random innovations $\epsilon_{j},-\infty<j<\infty$,

with values in a measurable space $S$, 
$X_{j}(t)=X_{j}(t, \omega)$ is jointly measurable in $(t, \omega) \quad(-\infty<j<\infty)$,

and

the sequence $\mathbf{X}$ can be approximated by $\ell$-dependent sequences $\left\{X_{j, \ell}\right\}_{j=-\infty}^{\infty}$ in the sense that

$$
\left(E\left\|X_{j}-X_{j, \ell}\right\|^{4}\right)^{1 / 4}=O\left(\ell^{-\alpha}\right)
$$

where $X_{j, \ell}$ is defined by $X_{j, \ell}=g\left(\epsilon_{j}, \epsilon_{j-1}, \ldots, \epsilon_{j-\ell+1}, \boldsymbol{\epsilon}_{j, \ell}^{*}\right)$,

$\boldsymbol{\epsilon}_{j, \ell}^{*}=\left(\epsilon_{j, \ell, j-\ell}^{*}, \epsilon_{j, \ell, j-\ell-1}^{*}, \ldots\right)$, where the $\epsilon_{j, \ell, k}^{*}$ 's are independent copies of $\epsilon_{0}$, independent of $\left\{\epsilon_{j},-\infty<j<\infty\right\}$.

This condition is a functional version of the assumption used by [15]. For a discussion of this assumption and its applications we refer to [11].

Theorem 2.2. If $\left\{X_{i}(t),-\infty<i<\infty, t \in[0,1]\right\}$ is an $L^{4}-m$-approximable Bernoulli shift with rate $\alpha>4$, then (2.2) and (2.3) hold.

Theorem 2.1 justifies the approximation

$E\left\|\hat{C}_{n, h}-C\right\|^{2} \approx \frac{h}{n}\left(\|C(t, s)\|^{2}+\left(\int C(t, t) d t\right)^{2}\right) \int_{-c}^{c} K^{2}(t) d t+\left\|E \hat{C}_{n, h}-C\right\|^{2}$,

for large $n$ which gives a significant simplification of how the integrated mean squared error depends on $h$. In case of the flat-top kernel $K_{f}(t ; x)$ of $(1.7)$ it is simple to get an upper bound for $\left\|E \hat{C}_{n, h}-C\right\|$.

Proposition 2.1. If (1.1), (1.7), (2.1) hold, $E\left\|X_{0}\right\|^{2}<\infty$, and

$$
\sum_{\ell=1}^{\infty} \ell\left\|a_{\ell}\right\|<\infty
$$

then

$$
\left\|E \hat{C}_{n, h}-C\right\|=O\left(\sum_{\ell=\lfloor h x\rfloor+1}^{\infty}\left\|a_{\ell}\right\|+\frac{h}{n} \sum_{\ell=1}^{\infty} \ell\left\|a_{\ell}\right\|\right)
$$


Comparing (2.9) to (2.4) it is clear that $(h / n) \sum_{\ell=1}^{\infty} \ell\left\|a_{\ell}\right\|$ has no asymptotic role in the exact order of the integrated mean squared error.

Remark 2.1. If $X_{i}(t)$ is a functional ARMA process then $\left\|a_{\ell}\right\|$ decreases exponentially fast as $\ell \rightarrow \infty$ (cf. [11] Ch. 13).

\section{Adaptive bandwidth selection}

\subsection{Bandwidth selection procedure}

One motive for using the flat-top kernel in (1.7) is that if the time series is uncorrelated after some lag $m$, then the kernel covariance estimators with bandwidths $h \geq\lceil m / x\rceil$ have negligible bias, where $\lceil y\rceil$ denotes the first integer larger than $y$. It then follows from Proposition 2.1 that the smallest bandwidth larger than $\lceil m / x\rceil$ gives the asymptotically smallest integrated mean squared error in this case.

This justifies the notion that the bandwidth should be chosen so that only the estimators for autocovariance terms at lags which appear to be significantly different from zero should be used in order to get the best possible rate of approximation. The autocovariance at lag $i$ is estimated by the function $\hat{\gamma}_{i}(t, s)$, and hence we have evidence that $\operatorname{Cov}\left(X_{0}(t), X_{i}(s)\right)$ is significantly different from the zero function if $\left\|\hat{\gamma}_{i}\right\|$ is large. To perform a hypothesis test in this direction we use the normalized statistic

$$
\hat{\rho}_{i}=\frac{\left\|\hat{\gamma}_{i}\right\|}{\int \hat{\gamma}_{0}(t, t) d t}
$$

which defines a functional analog of the autocorrelation. In fact, it follows from the Cauchy-Schwarz inequality that $0 \leq \hat{\rho}_{i} \leq 1$. We now explicitly define our adaptive bandwidth selection procedure:

Procedure for choosing $h$ : Find the first non-negative integer $\hat{m}$ such that $\hat{\rho}_{\hat{m}+r}<T \sqrt{\log n / n}$ for $r=1, \ldots, H$, where $T>0$, and $H$ is a positive integer. Take $h=\hat{h}$ where $\hat{h}=\lceil\hat{m} / x\rceil$.

This procedure is similar to the one given in [16] and describes a functional adaptation of choosing the bandwidth by inspecting the correlogram; we simply select $h$ so that the flat-top kernel estimator gives full weight to those autocovariances which are deemed to be significantly different from zero as a 
result of the comparison of the $\hat{\rho}_{i}^{\prime} s$ to $T \sqrt{\log n / n}$. The procedure terminates when a string of autocovariances of length $H$ cannot be distinguished from zero.

The following proposition shows that our procedure produces a bandwidth which adapts to the underlying level of dependence within the time series.

Proposition 3.1. If $\left\{X_{i}(t),-\infty<i<\infty, t \in[0,1]\right\}$ is $m$-dependent such that $\left\|\operatorname{Cov}\left(X_{0}(t), X_{i}(s)\right)\right\|>0$ for $1 \leq i \leq m$, then

$$
\lim _{n \rightarrow \infty} P(\hat{m}=m)=1,
$$

and hence the estimated bandwidth is $\lceil m / x\rceil$ with probability tending to 1.

\subsection{Implementation}

The bandwidth selection procedure may be implemented for a given functional time series upon the choice of the parameters $T$ and $H$. Although $T$ and $H$ can be chosen almost arbitrarily in order for the asymptotic result above to hold, we show by means of simulation in Section 4 that their choice can greatly affect the behavior of the bandwidth estimator for finite samples. The $\log n$, which we take to be the base 10 logarithm, appearing in the threshold in the definition of the procedure is needed to prove Proposition 3.1, however for practical sample sizes it is effectively a constant. This suggests taking $T$ to be a large quantile of the asymptotic distribution of $\sqrt{n} \hat{\rho}_{i}$ so that the threshold effectively represents a high coverage confidence interval assuming zero correlation at lag $i$.

For the scalar case considered in [16] the role of $\hat{\rho}_{i}$ is replaced by the simple autocorrelation of the time series which is known to have an asymptotic normal distribution under mild conditions. This motivates his choice of taking $T$ to be a suitably large quantile of the standard normal. The asymptotic distribution of $\hat{\rho}_{i}$ is more complicated though due to the infinite dimensional nature of functional data. It follows from the central limit theorem for finite dependent random functions (cf. [11], p. 297 and [4]) and the Karhunen-Loéve expansion that if $\left\{X_{i}(t),-\infty<i<\infty, t \in[0,1]\right\}$ is an $m$-dependent sequence then for $j>m$ 


$$
\sqrt{n} \hat{\rho}_{j} \stackrel{D}{\rightarrow} \frac{\left(\sum_{\ell=1}^{\infty} \lambda_{\ell, j} \chi_{\ell}^{2}(1)\right)^{1 / 2}}{\int E X_{0}^{2}(t) d t}
$$

where the $\lambda_{\ell, j}^{\prime} s$ are the eigenvalues of the asymptotic covariance operator of $\hat{\gamma}_{j}(t, s)$ and the $\chi_{\ell}^{2}(1)^{\prime} s$ are iid chi-square one random variables. An outline of the proof of (3.1) is included in Section 5.

The result in (3.1) can provide some insight for the choice of $T$. If, for example, the time series $\left\{X_{i}(t),-\infty<i<\infty, t \in[0,1]\right\}$ is iid with known covariance function $\operatorname{Cov}\left(X_{0}(t), X_{0}(s)\right)$, then the distribution on the right hand side of (3.1) is the same for all $j \geq 1$ and can be simulated. We performed this simulation over a broad collection of processes which included the Brownian motion, Brownian bridge, Ornstein-Uhlenbeck as well as several other non-normal processes and observed that the $90 \%$ to $99 \%$ quantiles of the right hand side of (3.1) never fell outside the interval $[1,3]$. We therefore investigate these values for $T$ in the simulation study below.

The main concern in choosing $H$ is that it should be large enough to give convincing evidence that all significant autocovariance estimators are used in $\hat{C}_{n, h}$. If, $\left\|\operatorname{Cov}\left(X_{0}(t), X_{i}(s)\right)\right\|$ is non-increasing in $i$ then the simple choice of $H=1$ would yield the sought after results. Although this is a common feature in real data, $\left\|\operatorname{Cov}\left(X_{0}(t), X_{i}(s)\right)\right\|$ need not be monotone and thus a larger value of $H$ may be desired. We noticed in the course of our own simulations that large choices of $H(\geq 6)$ tend to lead to high variance in the estimated bandwidths and overall poor estimation, and thus we recommend taking $H=3,4,5$.

\section{Simulation Study}

\subsection{Outline}

The goal of our simulation study is to investigate the bandwidth selection procedure proposed above as well as give practical advice on how to choose the bandwidth parameter $h$ to minimize $\left\|\hat{C}_{n, h}-C\right\|^{2}$ when using the flat-top kernel. All of the simulations in this section were performed using the $\mathrm{R}$ programming language. Below we use the kernel 


$$
K_{f}(t ; .5)=\left\{\begin{array}{lr}
1, & 0 \leq|t|<.5 \\
2-2|t|, & .5 \leq|t|<1 \\
0, & |t| \geq 1
\end{array}\right.
$$

to calculate all of the covariance estimators. By fixing the kernel throughout we hope to make the effects of changing the bandwidth more lucid. Using the kernel in (4.1) we compared our adaptive bandwidth selection procedure over the choices of the parameters $T$ and $H$ outlined in Section 3.2 to several fixed bandwidths using simulated data from a collection of data generating processes (DGP's) with varying degrees of dependence. In the following definitions we assume that $\left\{W_{i}(t),-\infty<i<\infty, t \in[0,1]\right\}$ are independent, identically distributed standard Brownian motions on $[0,1]$. The functional time series used for the simulation study follow either of the following models:

$$
\begin{array}{ll}
\operatorname{MA}_{\psi}(p): & X_{i}(t)=W_{i}(t)+\sum_{j=1}^{p} \int \psi(t, s) W_{i-j}(s) d s \\
\operatorname{FAR}_{\psi}(1): & X_{i}(t)=\int \psi(t, s) X_{i-1}(s) d s+W_{i}(t) \\
\operatorname{MA}_{\phi}^{*}(p): & X_{i}(t)=W_{i}(t)+\phi \sum_{j=1}^{p} W_{i-j}(t) \\
\operatorname{FAR}_{\phi}^{*}(1): & X_{i}(t)=\phi X_{i-1}(t) d s+W_{i}(t) .
\end{array}
$$

Specifically we considered the processes $\mathrm{MA}_{1}^{*}(0), \mathrm{MA}_{.5}^{*}(1), \mathrm{MA}_{.5}(4), \mathrm{MA}_{\psi_{1}}(4)$, $\mathrm{MA}_{.5}^{*}(8), \mathrm{FAR}_{.5}^{*}(1)$, and $\mathrm{FAR}_{\psi_{2}}(1)$, where $\psi_{1}(t, s)=.34 \exp \left(.5\left(t^{2}+s^{2}\right)\right)$ and $\psi_{2}(t, s)=3 / 2 \min (t, s)$.

The pointwise functional processes $\operatorname{MA}_{\phi}^{*}(p)$ and $\operatorname{FAR}_{\phi}^{*}(1)$ are used in [14] to model intraday price curves where it is argued that using these simpler models gives similar prediction results when compared to the more complicated $\mathrm{FAR}_{\psi}(1)$ model. In our application they possess the advantage that their long run covariance functions can be calculated explicitly. Figure 4.1 shows lattice plots of long run covariance kernel estimators with simulated $\mathrm{FAR}_{.5}^{*}(1)$ data using the adaptive bandwidth selection procedure taking $H=3$ and $T=2.0$ for $n=100,300$, and 500 as well as the theoretical long run covariance. 

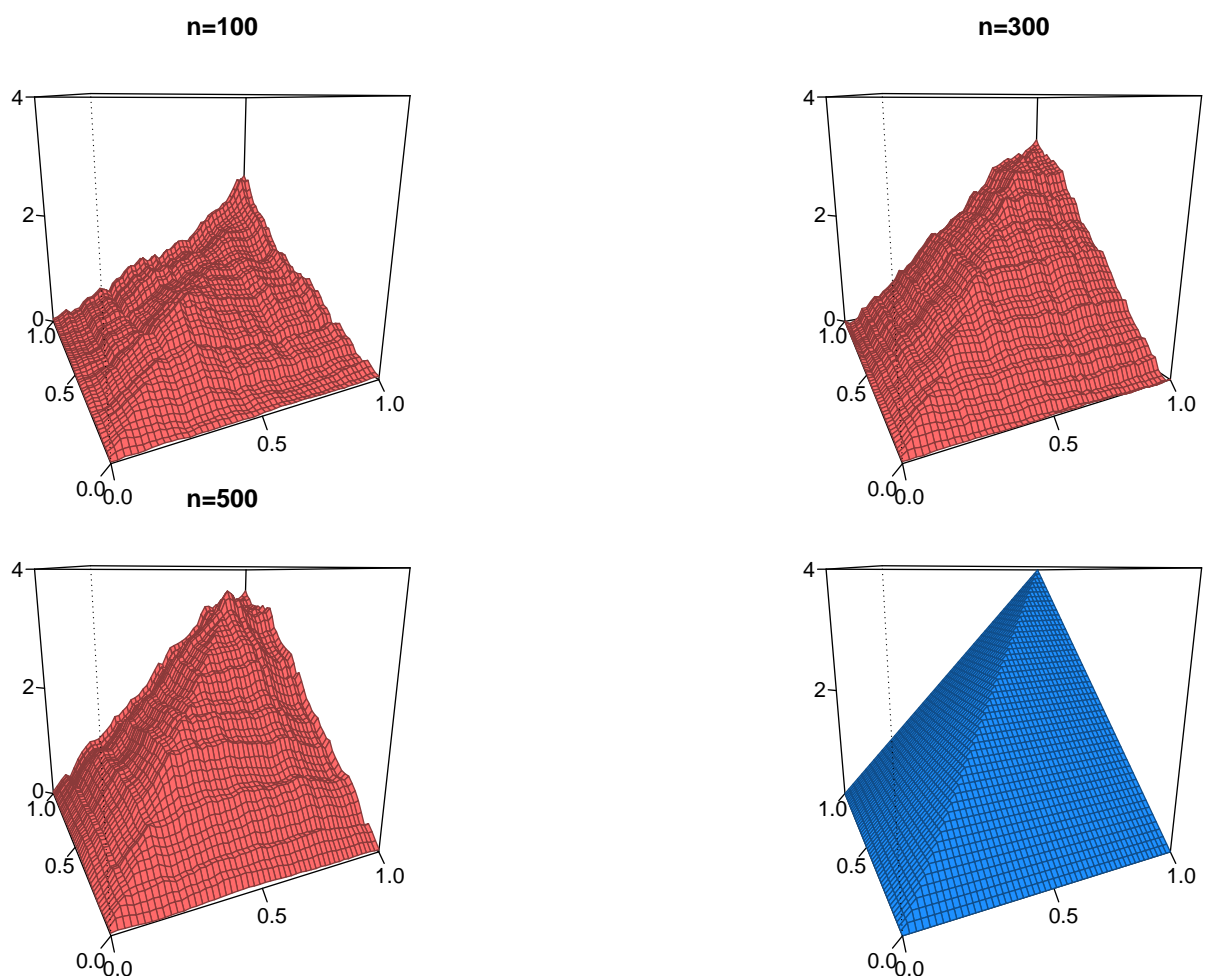

Figure 4.1: Lattice plots of the long run covariance kernel estimators with $\mathrm{FAR}_{5}^{*}(1)$ data using the adaptive bandwidth for values of $n=100,300$, and 500 along with the theoretical long run covariance (lower right). 
When the kernels $\psi_{1}$ and $\psi_{2}$ are used to define the process then it is not tractable to compute $C$ explicitly. In these cases $C$ is replaced by the approximation

$$
C^{*}(t, s)=\frac{1}{10^{4}} \sum_{j=1}^{10^{4}} \bar{X}_{j}(t) \bar{X}_{j}(s)
$$

where

$$
\bar{X}_{j}(t)=\frac{1}{10^{4}} \sum_{i=1}^{10^{4}} X_{i}^{(j)}(t),
$$

and the $X_{i}^{(j)}(t)^{\prime} s$ are computed according to data generating process $\mathrm{MA}_{\psi_{1}}(4)$ or $\mathrm{FAR}_{\psi_{2}}(1)$, independently for each $j$. This utilizes the fact that $C$ is the limiting covariance of the average of these processes. Since the norms $\left\|\psi_{1}\right\|,\left\|\psi_{2}\right\| \approx .5$, we expect the behavior of the bandwidth estimator to be roughly the same for the processes $\mathrm{MA}_{.5}^{*}(4)$ and $\mathrm{MA}_{\psi_{1}}(4)$ as well as for $\operatorname{FAR}_{.5}^{*}(1)$ and $\mathrm{FAR}_{\psi_{2}}(1)$.

In each iteration of the Monte Carlo simulation we approximate $L_{n, h}=$ $\left\|\hat{C}_{n, h}-C\right\|^{2}$ for a particular bandwidth choice $h$ by a simple Riemann sum approximation. Each simulation was repeated independently 1000 times for each DGP with values of $n=100,200,300$, and 500. The values of $\bar{L}_{n, h}$ and $\tilde{L}_{n, h}$ are reported for $h=\hat{h}$ using $T=1,1.5,2,2.5,3$ and $H=3,4,5$ as well as the fixed bandwidths $h=n^{1 / 4}, n^{1 / 2}$ where $\bar{L}_{n, h}$ denotes the mean of $L_{n, h}$ over the 1000 simulations and $\tilde{L}_{n, h}$ denotes the median. For comparison these summary statistics are also given for $h=h_{\text {opt }}$, where

$$
h_{o p t}=\underset{h}{\operatorname{argmin}} L_{n, h} .
$$

\subsection{Results}

The summary statistics $\bar{L}_{n, h}$ and $\tilde{L}_{n, h}$ for all simulations performed are provided in Tables 4.1-4.14 which we summarize as follows:

1. Over all bandwidth choices the accuracy of the estimation improves by increasing $n$, as expected.

2. Also as expected, the estimation accuracy decreases by increasing the level of dependence.

3. In terms of choosing the tuning parameters in the adaptive bandwidth procedure we see that in general the best results are obtained for $T=$ 2.0,2.5 and $H=3$ for the DGP's we considered. 


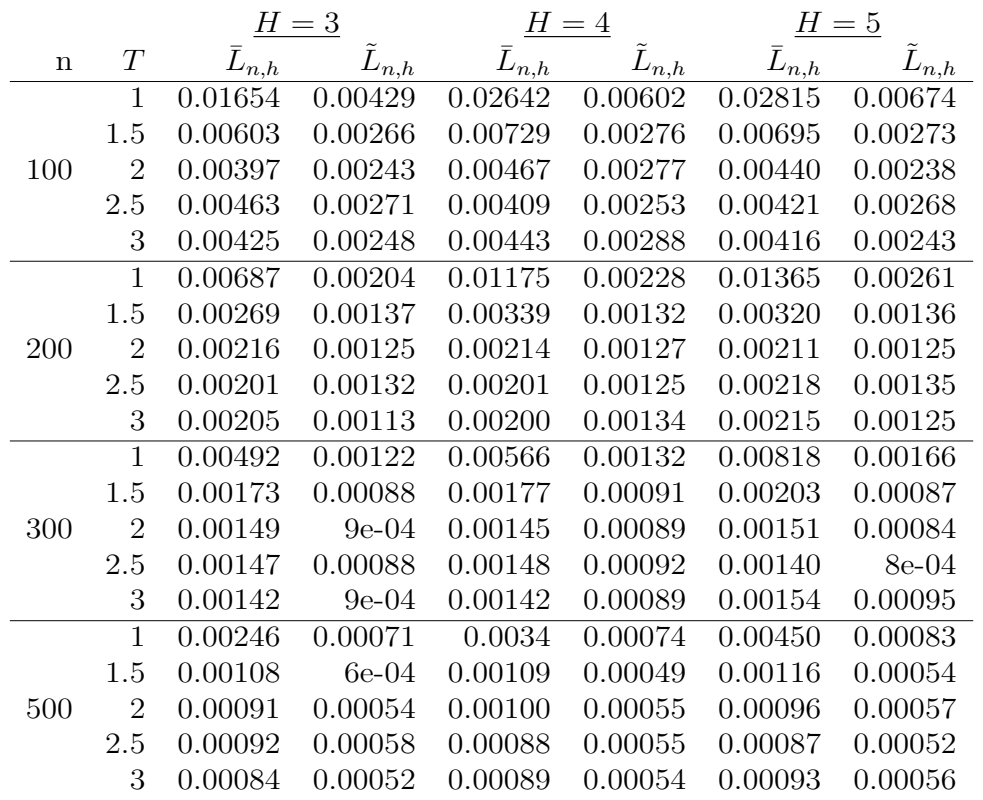

Table 4.1: Results for $\mathrm{MA}_{1}^{*}(0)$ with estimated bandwidths.

\begin{tabular}{|c|c|c|c|c|c|c|c|c|}
\hline & \multicolumn{2}{|c|}{$n=100$} & \multicolumn{2}{|c|}{$n=200$} & \multicolumn{2}{|c|}{$n=300$} & \multicolumn{2}{|c|}{$n=500$} \\
\hline & $\overline{\bar{L}_{n, h}}$ & $\tilde{L}_{n, h}$ & $\overline{\bar{L}_{n, h}}$ & $\tilde{L}_{n, h}$ & $\overline{\bar{L}}_{n, h}$ & $\tilde{L}_{n, h}$ & $\overline{\bar{L}_{n, h}}$ & $\tilde{L}_{n}$ \\
\hline & & 0.002 & & 0.00 & 108 & & & \\
\hline & $48 / 3$ & 032 & 3800 & 20 & 3095 & 0.01 & 02423 & \\
\hline & 190 & & & 0682 & 090 & 00542 & 00558 & \\
\hline
\end{tabular}

Table 4.2: Results for $\mathrm{MA}_{1}^{*}(0)$ with fixed bandwidths. 


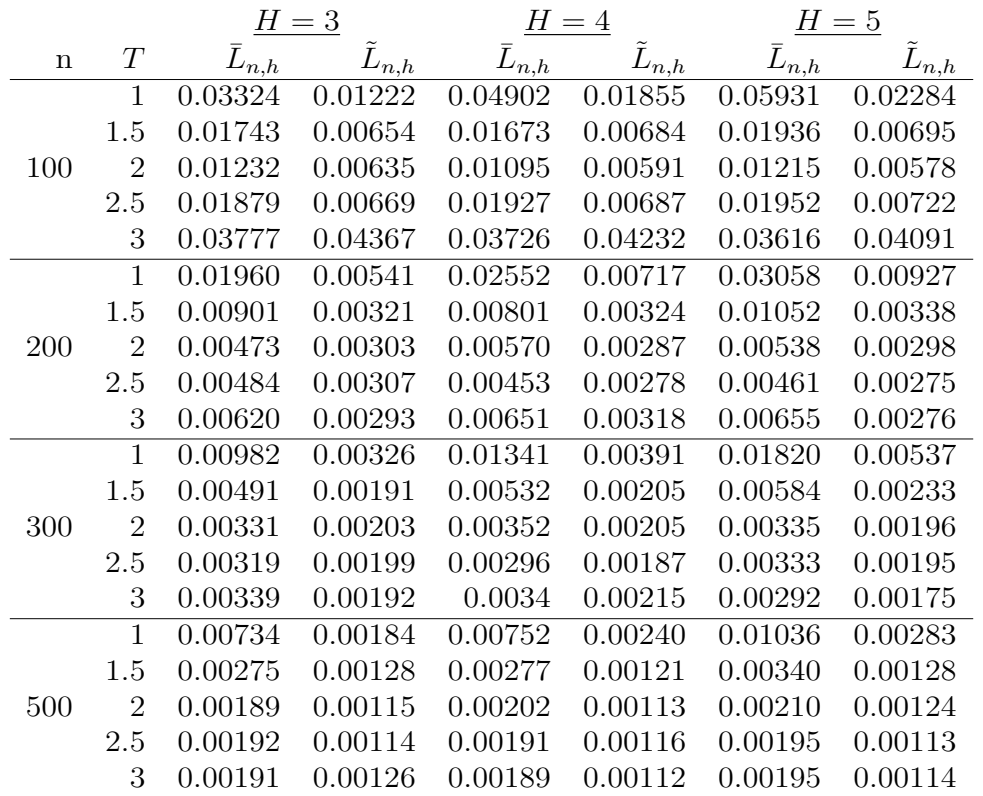

Table 4.3: Results for $\mathrm{MA}_{.5}^{*}(1)$ with estimated bandwidths.

\begin{tabular}{|c|c|c|c|c|c|c|c|c|}
\hline & \multicolumn{2}{|c|}{$n=100$} & \multicolumn{2}{|c|}{$n=200$} & \multicolumn{2}{|c|}{$n=300$} & \multicolumn{2}{|c|}{$n=500$} \\
\hline & $\overline{\bar{L}_{n, h}}$ & $\tilde{L}_{n, h}$ & $\overline{\bar{L}_{n, h}}$ & $\tilde{L}_{n, h}$ & $\overline{\bar{L}}_{n, h}$ & $\tilde{L}_{n, h}$ & $\overline{\bar{L}_{n, h}}$ & $\tilde{L}_{n,}$ \\
\hline & 01 & .004 & & 0.002 & 257 & 0. & & \\
\hline & $4 \| 11$ & 31 & 3786 & 13 & 3048 & 0.01 & 02447 & \\
\hline & 2002 & 7 & 036 & & & & 0 & \\
\hline
\end{tabular}

Table 4.4: Results for MA.5(1) with fixed bandwidths. 


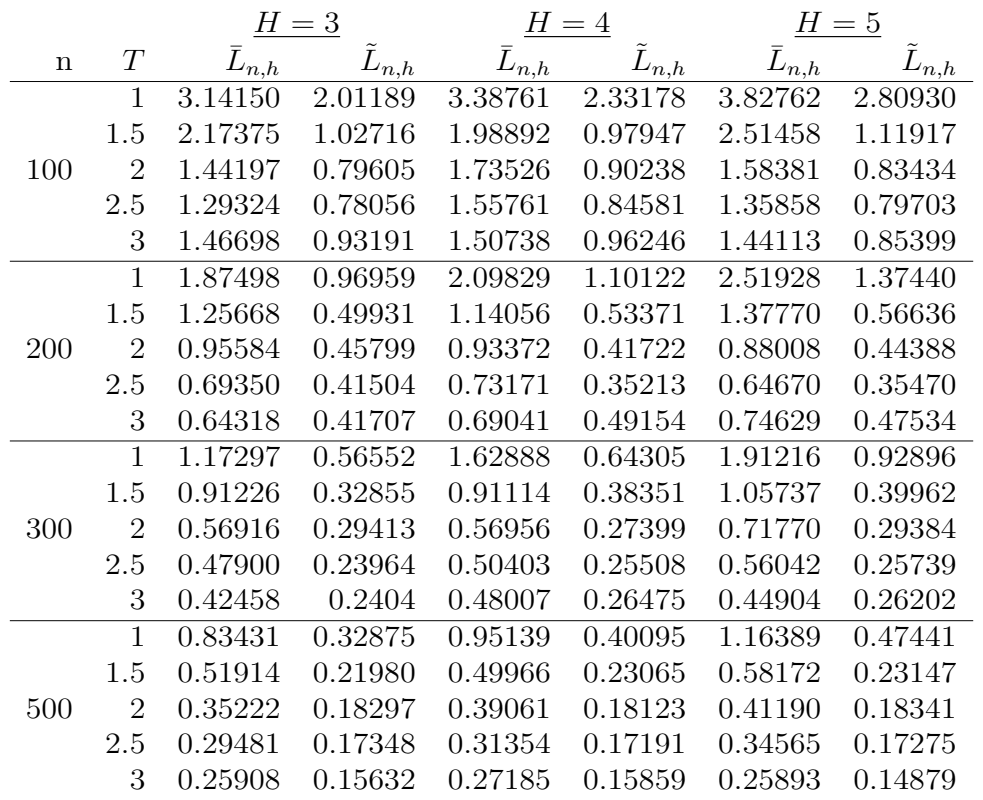

Table 4.5: Results for $\mathrm{MA}_{.5}^{*}(4)$ with estimated bandwidths.

\begin{tabular}{|c|c|c|c|c|c|c|c|c|}
\hline & \multicolumn{2}{|c|}{$l=100$} & \multicolumn{2}{|c|}{$n=200$} & \multicolumn{2}{|r|}{$n=300$} & \multicolumn{2}{|c|}{$\underline{n=500}$} \\
\hline & $\bar{L}_{n, h}$ & $\tilde{L}_{n, h}$ & $\overline{\bar{L}_{n, h}}$ & $\tilde{L}_{n, h}$ & $\bar{L}_{n, h}$ & $\tilde{L}_{n, h}$ & $\bar{L}_{n, h}$ & $\tilde{L}_{n}$ \\
\hline & & 0.373 & & 0.18 & 24 & 0.122 & & \\
\hline & 5665 & 84 & & 0928 & & 0.74392 & 92265 & 0.59 \\
\hline & & & & & 40 & 0.22979 & 235558 & \\
\hline
\end{tabular}

Table 4.6: Results for MA.5(4) with fixed bandwidths. 


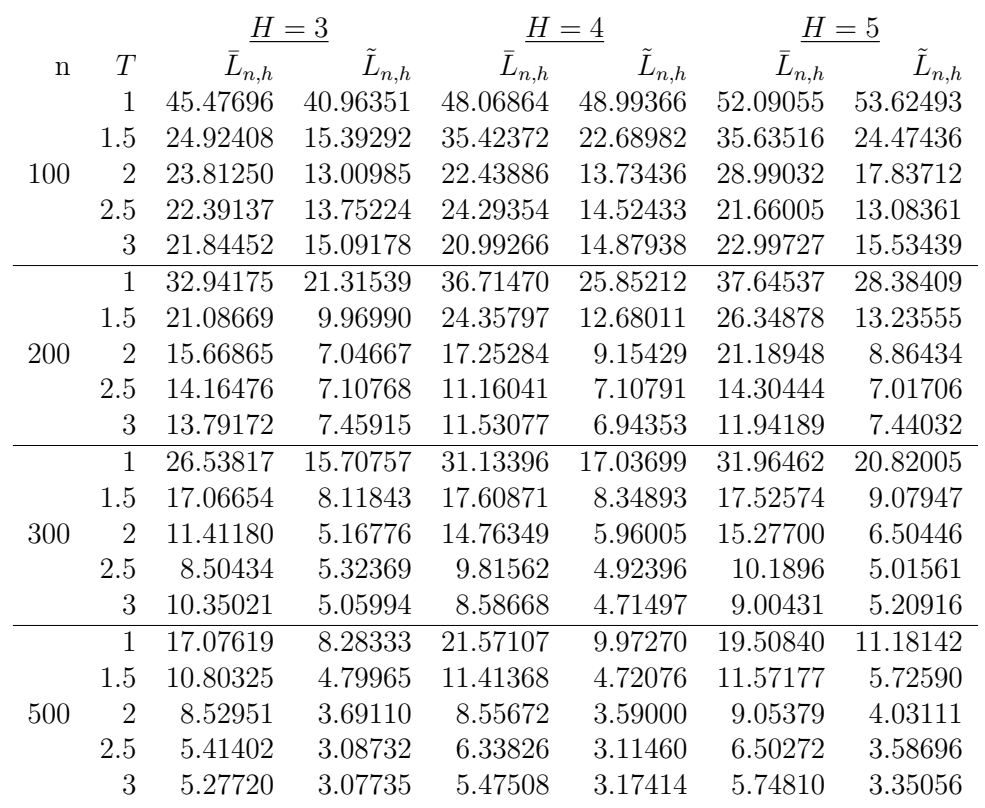

Table 4.7: Results for $\mathrm{MA}_{.5}^{*}(8)$ with estimated bandwidths.

\begin{tabular}{|c|c|c|c|c|c|c|c|c|}
\hline \multirow[t]{2}{*}{$h$} & \multicolumn{2}{|c|}{$\underline{n=100}$} & \multicolumn{2}{|c|}{$n=200$} & \multicolumn{2}{|c|}{$n=300$} & \multicolumn{2}{|c|}{$n=500$} \\
\hline & $\bar{L}_{n, h}$ & $\tilde{L}_{n, h}$ & $\bar{L}_{n, h}$ & $\tilde{L}_{n, h}$ & $\bar{L}_{n, h}$ & $\tilde{L}_{n, l}$ & $\bar{L}_{n, h}$ & $\tilde{L}_{n, h}$ \\
\hline & & 7.718 & & & & & & \\
\hline $1 / 2$ & 84182 & & & & & 7.528 & 9.25654 & \\
\hline & & & & & & & 87572 & \\
\hline
\end{tabular}

Table 4.8: Results for $\mathrm{MA}_{.5}^{*}(8)$ with fixed bandwidths. 


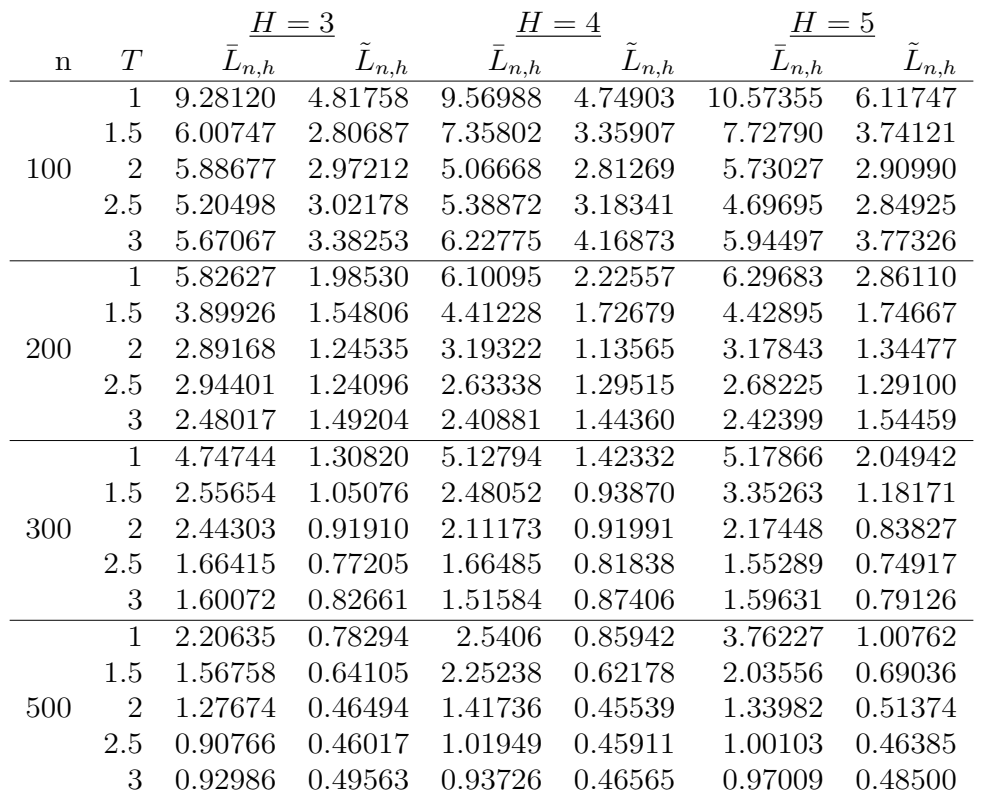

Table 4.9: Results for $\mathrm{MA}_{\psi_{1}}$ (4) with estimated bandwidths.

\begin{tabular}{|c|c|c|c|c|c|c|c|c|}
\hline & \multicolumn{2}{|c|}{$n=100$} & \multicolumn{2}{|c|}{$n=200$} & \multicolumn{2}{|c|}{$n=300$} & \multicolumn{2}{|c|}{$n=500$} \\
\hline & $\overline{\bar{L}_{n, h}}$ & $\tilde{L}_{n, h}$ & $\overline{\bar{L}_{n, h}}$ & $\tilde{L}_{n, h}$ & $\overline{\bar{L}_{n, h}}$ & $\tilde{L}_{n, h}$ & $\overline{\bar{L}}_{n, h}$ & $\tilde{L}_{n}$ \\
\hline & 0 & $\pi$ & 2809 & & 69879 & 0.11 & 42800 & \\
\hline & & & & & 77693 & & 84981 & 1.50 \\
\hline & & & 34030 & & 33308 & 82854 & 95135 & \\
\hline
\end{tabular}

Table 4.10: Results for $\mathrm{MA}_{\psi_{1}}(4)$ with fixed bandwidths. 


\begin{tabular}{|c|c|c|c|c|c|c|c|}
\hline \multirow[b]{2}{*}{$\mathrm{n}$} & \multicolumn{3}{|c|}{$H=3$} & \multicolumn{2}{|c|}{$H=4$} & \multicolumn{2}{|c|}{$H=5$} \\
\hline & $T$ & $\bar{L}_{n, h}$ & $\tilde{L}_{n, h}$ & $\bar{L}_{n, h}$ & $\tilde{L}_{n, h}$ & $\bar{L}_{n, h}$ & $\tilde{L}_{n, h}$ \\
\hline \multirow{5}{*}{100} & 1 & 0.76698 & 0.42362 & 0.93932 & 0.49948 & 1.03109 & 0.56456 \\
\hline & 1.5 & 65751 & 0.39061 & .64588 & 0.44584 & 0.77110 & 0.43790 \\
\hline & 2 & 0.54772 & 0.42784 & 0.55678 & 0.42740 & 0.54312 & 0.42640 \\
\hline & 2.5 & 0.66762 & 0.44354 & 0.72712 & 0.46846 & 0.66582 & 0.43636 \\
\hline & 3 & 0.95361 & 1.21976 & & 1.21411 & 0.94428 & 1.22441 \\
\hline \multirow{5}{*}{200} & 1 & .39446 & & 58 & & 0.72 & 0.28331 \\
\hline & 1.5 & & & & & & \\
\hline & 2 & & & & & & \\
\hline & 2.5 & & & & & & 0.38290 \\
\hline & 3 & & & & & & 0.38817 \\
\hline \multirow{5}{*}{300} & 1 & & & & & & \\
\hline & 1.5 & & & & & & 0.1 \\
\hline & 2 & 644 & 0.1 & 0.25736 & 229 & 0.23917 & 0.13510 \\
\hline & 2.5 & & & & & 41 & 0.33062 \\
\hline & 3 & & & & & 197 & 0.34855 \\
\hline \multirow{5}{*}{500} & 1 & & & & & & 0.11960 \\
\hline & 1.5 & & 0.08683 & & & 0.15914 & 0.09552 \\
\hline & 2 & 0.14349 & 0.0937 & .15890 & 0.09540 & 0.14368 & 0.08867 \\
\hline & 2.5 & 0.18816 & 0.09211 & .19216 & 0.10440 & 0.19805 & 0.10497 \\
\hline & 0 & 0.27474 & 0.30473 & 0.27174 & 0.30522 & 0.28638 & 0.31384 \\
\hline
\end{tabular}

Table 4.11: Results for $\mathrm{FAR}_{.5}^{*}(1)$ with estimated bandwidths.

\begin{tabular}{|c|c|c|c|c|c|c|c|c|}
\hline & \multicolumn{2}{|c|}{$=100$} & \multicolumn{2}{|c|}{$n=200$} & \multicolumn{2}{|c|}{$n=300$} & \multicolumn{2}{|c|}{$n=500$} \\
\hline & $\overline{\bar{L}_{n, h}}$ & $\tilde{L}_{n, h}$ & $\overline{\bar{L}_{n, h}}$ & $\tilde{L}_{n, h}$ & $\bar{L}_{n,}$ & $\tilde{L}_{n, h}$ & $\bar{L}_{n, h}$ & $\tilde{L}_{n, l}$ \\
\hline & & 0.141 & & 0.07216 & 9963 & 0.05 & 06423 & \\
\hline & & & & & 8430 & 0.30829 & 37941 & \\
\hline & 3869 & 240 & 19782 & 13559 & 14958 & 0.098 & 09951 & 16 \\
\hline
\end{tabular}

Table 4.12: Results for FAR.5(1) with fixed bandwidths. 


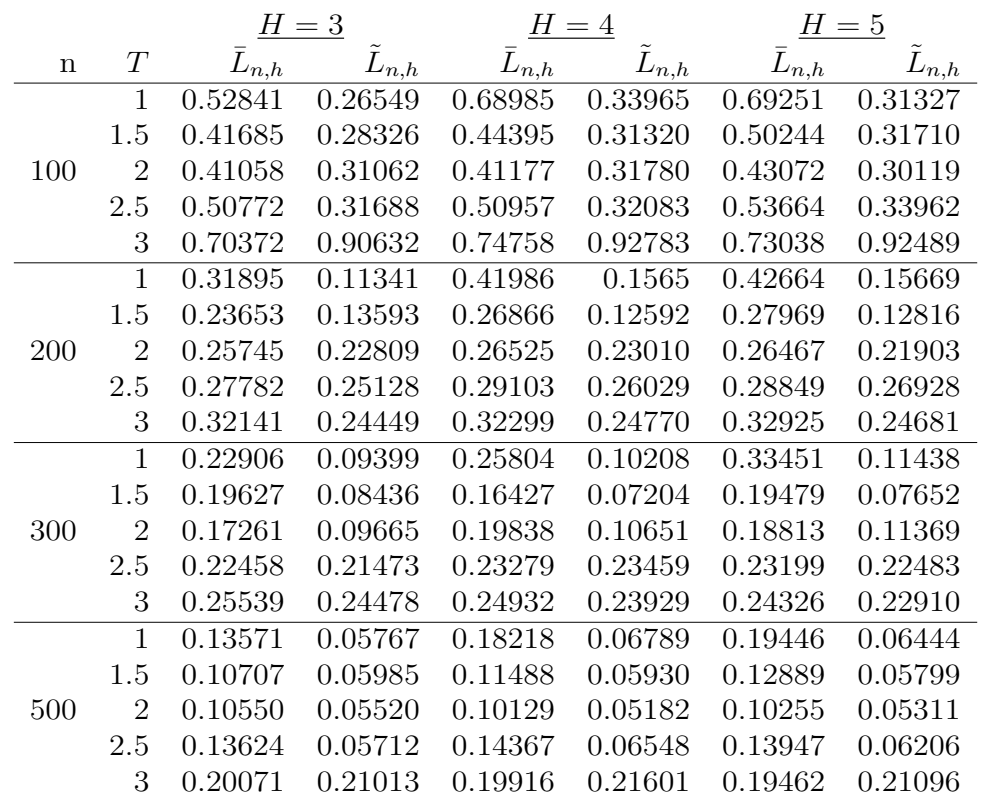

Table 4.13: Results for $\mathrm{FAR}_{\psi_{2}}(1)$ with estimated bandwidths.

\begin{tabular}{|c|c|c|c|c|c|c|c|c|}
\hline & \multicolumn{2}{|c|}{$n=100$} & \multicolumn{2}{|c|}{$n=200$} & \multicolumn{2}{|c|}{$n=300$} & \multicolumn{2}{|c|}{$n=500$} \\
\hline & $\overline{\bar{L}_{n, h}}$ & $\tilde{L}_{n, h}$ & $\overline{\bar{L}_{n, h}}$ & $\tilde{L}_{n, h}$ & $L_{n,}$ & $\tilde{L}_{n, h}$ & $\overline{\overline{L_{n, h}}}$ & $\tilde{L}_{n, l}$ \\
\hline & 4 & 4.00 & 7254 & & 1842 & & & .000 \\
\hline & 0754 & 968 & & & 39 & 0.18029 & .2754 & 0.13 \\
\hline & 3160 & $1 x_{2}$ & 13083 & & 961 & & 06288 & 3 \\
\hline
\end{tabular}

Table 4.14: Results for $\mathrm{FAR}_{\psi_{2}}(1)$ with fixed bandwidths. 
4. Comparing our adaptive bandwidth to the commonly used fixed bandwidth of $n^{1 / 4}$ and $n^{1 / 2}$ we see that:

(a) Our adaptive bandwidth outperforms the fixed bandwidths when the dependence is weak $\left(\mathrm{MA}_{1}^{*}(0), \mathrm{MA}_{.5}^{*}(1)\right)$

(b) When the dependence is moderate $\left(\mathrm{MA}_{.5}^{*}(4), \mathrm{MA}_{\psi_{1}}(4) \mathrm{FAR}_{.5}^{*}(1)\right.$, $\left.\operatorname{FAR}_{\psi_{2}}(1)\right)$ the bandwidth $h=n^{1 / 4}$ performs the best for values of $n \leq 200$, however for $n \geq 300$ the adaptive bandwidth gives nearly equivalent accuracy.

(c) When the dependence is strong $\left(\mathrm{MA}_{.5}^{*}(8)\right)$ the adaptive bandwidth again gives the best accuracy among the bandwidths considered.

In conclusion we recommend that if weak dependence is suspected in the functional time series, or if the sample size is large $(n \geq 300)$ the adaptive bandwidth should be used in the estimation of the long run covariance function. If the sample size is small $(n<300)$ and moderate dependence is expected, then $h=n^{1 / 4}$ may be preferable.

\subsection{Application to cumulative intraday returns data}

In order to illustrate the applicability of our method we considered the problem of estimating the long run covariance of a functional time series derived from asset price data. The functional time series we considered was constructed from one-minute resolution price of Citigroup stock over a ten year period from April 1997, to April 2007 comprising 2511 days. In each day there are 390 recordings of the stock price, corresponding to a 390 minute trading day, which we linearly interpolated to obtain daily price curves. Since asset price data rarely appears stationary, we work instead with a functional version of the log returns which are defined as follows.

Definition 4.1. Suppose $P_{j}(t)$ is the price of an asset at time $t$ on day $j$ for $t \in[0,1], j=1, \ldots, n$. The functions $R_{j}(t)=100\left(\ln P_{j}(t)-\ln P_{j}(0)\right), t \in$ $[0,1], j=1, \ldots, n$, are called the cumulative intraday return (CIDR) curves.

The first week ( 5 days) of CIDR curves derived from the Citigroup price data are shown in Figure 4.2. The stationarity of the CIDR curves is investigated in detail in [10].

Before turning to the estimation of the long run covariance of the CIDR curves, we first illustrate another possible use of the adaptive bandwidth 


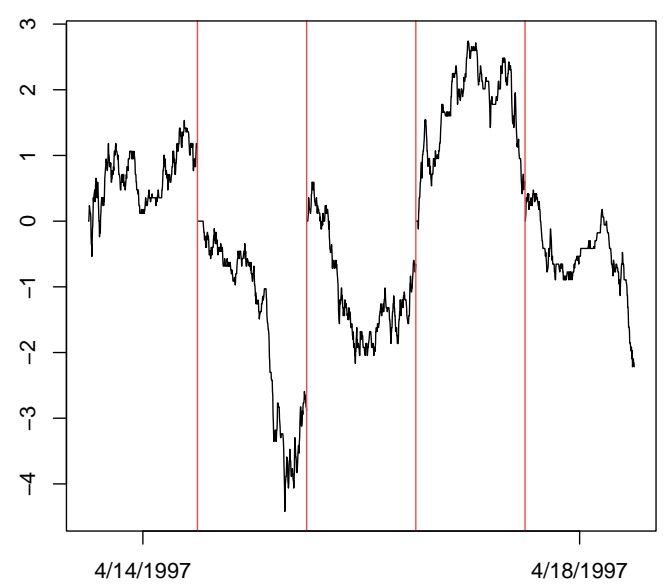

Figure 4.2: Cumulative intraday return curves derived from one minute resolution Citigroup stock price.

estimator: to assess the level of dependence in the sequence. If, for example, the values of $\hat{m}$ are small when computed over several subsegments of the sample it indicates that the autocorrelation is likely negligible for larger lags. Conversely if $\hat{m}$ tends to be large when computed on subsegments it indicates that the time series exhibits strong serial correlation. Summary statistics for $\hat{m}$ computed from subsegments of various lengths of the CIDR curves derived from the Citigroup stock price are given in Table 4.15. Over the three segment lengths considered nearly all of the $\hat{m}^{\prime} s$ computed were zero, which indicates that the sequence is apparently uncorrelated. This is the typical behavior of a functional ARCH sequence, see [7].

\begin{tabular}{cccc}
$n$ & \# of segments & mean $\hat{m}$ & median $\hat{m}$ \\
\hline 100 & 25 & 0.08 & 0 \\
200 & 12 & 0.17 & 0 \\
300 & 8 & 0 & 0
\end{tabular}

Table 4.15: Summary statistics for values of $\hat{m}$ computed from segments of lengths $n=$ 100, 200 and 300 of the CIDR curves derived from the Citigroup stock price. 


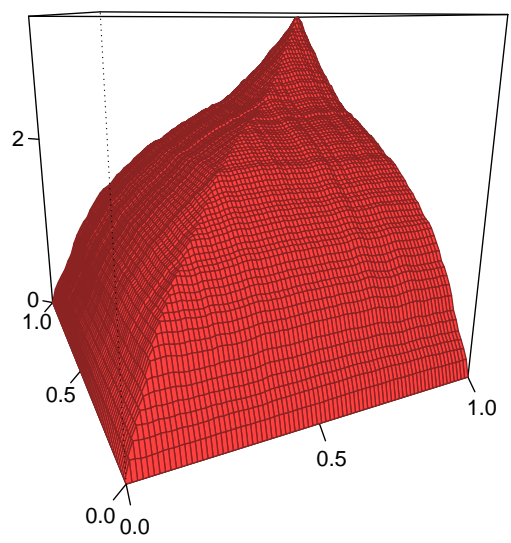

Figure 4.3: Lattice plot of the long run covariance kernel estimator of the CIDR curves derived from Citigroup stock price data using the flat-top kernel and adaptive bandwidth.

Given this, and the fact that the sample size is large, it is advisable to use the adaptive bandwidth estimator in order to compute the long run covariance estimator. A lattice plot of the long run covariance estimator based on the entire sample of Citigroup CIDR curves using the flat-top kernel $K_{f}(t ; .5), T=2.0$ and $H=3$ is shown in Figure 4.3.

Previously the CIDR curves have been compared to realizations of Brownian motions, which seems accurate based on their appearance in Figure 4.2. Figure 4.3 sheds more light on this comparison since the long run covariance of the CIDR curves appears to be a functional of $\min (t, s)$, the covariance function of the standard Brownian motion, which is consistent with the hypothesis that the CIDR curves behave according to a functional $\mathrm{ARCH}$ process based on Brownian motion errors.

\section{Proofs}

\subsection{Proof of Theorem 2.1}

By a simple calculation using stationarity we get 


$$
\begin{aligned}
& n \operatorname{Cov}\left(\hat{\gamma}_{\ell}(t, s), \hat{\gamma}_{g}(t, s)\right) \\
& =\frac{1}{n}\left(\sum_{i=\max \{1,1-\ell\}}^{\min \{n, n-\ell\}} \sum_{j=\max \{1,1-g\}}^{\min \{n, n-g\}} E X_{i}(t) X_{i+\ell}(s) X_{j}(t) X_{j+g}(s)\right. \\
& \left.\quad-(n-|\ell|)(n-|g|) a_{\ell}(t, s) a_{g}(t, s)\right) \\
& =\frac{1}{n} \sum_{i=\max \{1,1-\ell\}} \sum_{j=\max \{1,1-g\}}^{\min \{n, n-\ell\}}\left(\Psi_{\ell, j-i, j-i+g}(t, s)\right. \\
& \left.+a_{j-i+g}(t, s) a_{j-i-\ell}(t, s)+a_{j-i}(t, t) a_{j-i+g-\ell}(s, s)\right) .
\end{aligned}
$$

Notice that the summand in the last term only depends on the difference $j-i$. Let $\phi_{n}(r, \ell, g)=\mid\{(i, j): j-i=r, \max \{1,1-\ell\} \leq i \leq \min \{n, n-$ $\ell\}, \max \{1,1-g\} \leq j \leq \min \{n, n-g\}\} \mid$, i.e. $\phi_{n}$ denotes the number of pairs of indices $i, j$ in the sum so that $j-i=r$. Clearly for all $r, \ell$, and $g, \phi_{n}(r, \ell, g) \leq n$. Also $\phi_{n}(r, \ell, g) \geq n-2(|\ell|+|r|+|g|)$, since $\{(i, i+r)$ : $\max \{|r|, 1-\ell+|r|, 1-g+|r|\} \leq i \leq \min \{n-|r|, n-g-|r|, n-\ell-|r|\}\} \subseteq$ $\{(i, j): j-i=r, \max \{1,1-\ell\} \leq i \leq \min \{n, n-\ell\}, \max \{1,1-g\} \leq j \leq$ $\min \{n, n-g\}\}$. Hence if $\theta_{n}(r, \ell, g)=\phi_{n}(r, \ell, g) / n$,

$$
\begin{aligned}
& n \operatorname{Cov}\left(\hat{\gamma}_{\ell}(t, s), \hat{\gamma}_{g}(t, s)\right) \\
& \quad=\sum_{r=-(n-1)}^{n-1} \theta_{n}(r, \ell, g)\left[\Psi_{\ell, r, r+g}(t, s)+a_{r+g}(t, s) a_{r-\ell}(t, s)+a_{r}(t, t) a_{r+g-\ell}(s, s)\right] .
\end{aligned}
$$

It follows that

$$
\begin{aligned}
\frac{n}{h} \operatorname{Var}\left(\hat{C}_{n}(t, s)\right) & =\frac{n}{h} \sum_{g, \ell=-h}^{h} K\left(\frac{g}{h}\right) K\left(\frac{\ell}{h}\right) \operatorname{Cov}\left(\hat{\gamma}_{\ell}(t, s), \hat{\gamma}_{g}(t, s)\right) \\
& =q_{1, n}(t, s)+q_{2, n}(t, s)+q_{3, n}(t, s)
\end{aligned}
$$

where 


$$
\begin{gathered}
q_{1, n}(t, s)=\frac{1}{h} \sum_{g, \ell=-h}^{h} K\left(\frac{g}{h}\right) K\left(\frac{\ell}{h}\right) \sum_{r=-(n-1)}^{n-1} \theta_{n}(r, \ell, g) \Psi_{\ell, r, r+g}(t, s), \\
q_{2, n}(t, s)=\frac{1}{h} \sum_{g, \ell=-h}^{h} K\left(\frac{g}{h}\right) K\left(\frac{\ell}{h}\right) \sum_{r=-(n-1)}^{n-1} \theta_{n}(r, \ell, g) a_{r+g}(t, s) a_{r-\ell}(t, s),
\end{gathered}
$$

and

$$
q_{3, n}(t, s)=\frac{1}{h} \sum_{g, \ell=-h}^{h} K\left(\frac{g}{h}\right) K\left(\frac{\ell}{h}\right) \sum_{r=-(n-1)}^{n-1} \theta_{n}(r, \ell, g) a_{r}(t, t) a_{r+g-\ell}(s, s) .
$$

First we consider the limit of $\iint q_{2, n}(t, s) d t d s$. Let $\varepsilon>0$. By a change of variables

$$
\begin{aligned}
q_{2, n}(t, s)=\frac{1}{h} \sum_{|u|,|v| \leq h+n-1} \sum_{r=b_{1}(u, v, n)}^{b_{2}(u, v, n)} & K\left(\frac{u-r}{h}\right) K\left(\frac{v-r}{h}\right) \\
& \times \theta_{n}(r, r+u, v-r) a_{u}(t, s) a_{v}(t, s) .
\end{aligned}
$$

where $b_{1}(u, v, n)=\max \{u-h, v-h,-(n-1)\}$ and $b_{2}(u, v, n)=\min \{u+$ $h, v+h, n-1\}$. Let

$$
\begin{aligned}
q_{2, n}^{(m)}(t, s)=\frac{1}{h} \sum_{|u|,|v| \leq m} \sum_{r=b_{1}(u, v, n)}^{b_{2}(u, v, n)} & K\left(\frac{u-r}{h}\right) K\left(\frac{v-r}{h}\right) \\
& \times \theta_{n}(r, r+u, v-r) a_{u}(t, s) a_{v}(t, s) .
\end{aligned}
$$

Then with $D=\{(u, v): 0 \leq|u|,|v| \leq h+n-1, \max (|u|,|v|) \geq m\}$

$$
\begin{aligned}
& \left|q_{2, n}(t, s)-q_{2, n}^{(m)}(t, s)\right| \\
& \quad \leq \frac{1}{h} \sum_{D} \sum_{r=b_{1}(u, v, n)}^{b_{2}(u, v, n)} K\left(\frac{u-r}{h}\right) K\left(\frac{v-r}{h}\right) \theta_{n}(r, r+u, v-r)\left|a_{u}(t, s) a_{v}(t, s)\right| .
\end{aligned}
$$


Since $K(x)=0$ for $|x|>c$, the number of terms in $r$ such that $b_{1}(u, v, n) \leq$ $r \leq b_{2}(u, v, n)$ and $K((u-r) / h) K((v-r) / h) \neq 0$ cannot exceed $2 h c$ for all $u, v$. Furthermore $K((u-r) / h) K((v-r) / h) \leq \sup _{-c \leq x \leq c} K^{2}(x)$. Since $0 \leq \theta_{n} \leq 1$, it follows that

$$
\left|q_{2, n}(t, s)-q_{2, n}^{(m)}(t, s)\right| \leq 2 c \sup _{-c \leq x \leq c} K^{2}(x) \sum_{m \leq|u|,|v| \leq h+(n-1)}\left|a_{u}(t, s) a_{v}(t, s)\right| .
$$

Therefore by the Cauchy-Schwarz inequality

$$
\begin{aligned}
& \left|\iint q_{2, n}(t, s)-q_{2, n}^{(m)}(t, s) d t d s\right| \\
& \leq 2 c \sup _{-c \leq x \leq c} K^{2}(x) \sum_{m \leq|u|,|v| \leq h+(n-1)} \iint\left|a_{u}(t, s) a_{v}(t, s)\right| d t d s \\
& \leq 4 c \sup _{-c \leq x \leq c} K^{2}(x)\left(\sum_{m \leq|u|<\infty}\left\|a_{u}\right\|\right)\left(\sum_{v=-\infty}^{\infty}\left\|a_{v}\right\|\right)<\varepsilon / 4
\end{aligned}
$$

by taking $m$ sufficiently large according to (2.2). When $|u|,|v| \leq m, b_{1}(u, v, n) \leq$ $r \leq b_{2}(u, v, n)$ implies that $|r| \leq m+h$, and hence for such $u, r$, and $v$, $\left|\theta_{n}(r, r+u, v-r)-1\right| \leq 2(5 m+3 h) / n$. It follows along the lines of (5.1) that

$$
\begin{aligned}
& \mid \iint q_{2, n}^{(m)}(t, s) \\
& \quad-\frac{1}{h} \sum_{|u|,|v| \leq m} \sum_{r=b_{1}(u, v, n)}^{b_{2}(u, v, n)} K\left(\frac{u-r}{h}\right) K\left(\frac{v-r}{h}\right) a_{u}(t, s) a_{v}(t, s) d t d s \mid<\varepsilon / 4
\end{aligned}
$$

for $n$ sufficiently large. Since $K(x)$ is continuous with compact support, and $h \rightarrow \infty$ as $n \rightarrow \infty$ we get that for all $|u|,|v| \leq m$ and $\eta>0$

$$
\left|K\left(\frac{u-r}{h}\right) K\left(\frac{v-r}{h}\right)-K^{2}\left(\frac{r}{h}\right)\right|<\eta
$$


for all $r$ when $n$ is sufficiently large. We then obtain by taking $\eta$ sufficiently small that

$$
\begin{aligned}
\mid \iint \frac{1}{h} \sum_{|u|,|v| \leq m} \sum_{r=b_{1}(u, v, n)}^{b_{2}(u, v, n)}\left[K\left(\frac{u-r}{h}\right)\right. & K\left(\frac{v-r}{h}\right) \\
& \left.-K^{2}\left(\frac{r}{h}\right)\right] a_{u}(t, s) a_{v}(t, s) d t d s \mid<\varepsilon / 4
\end{aligned}
$$

for sufficiently large $n$. By the definition of the Riemann integral

$$
\frac{1}{h} \sum_{r=b_{1}(u, v, n)}^{b_{2}(u, v, n)} K^{2}\left(\frac{r}{h}\right) \rightarrow \int_{-c}^{c} K^{2}(t) d t
$$

as $n \rightarrow \infty$. Also by the definition of $C(t, s)$

$$
\begin{aligned}
\iint \sum_{|u|,|v| \leq m} a_{u}(t, s) a_{v}(t, s) d t d s & =\iint\left(\sum_{|u| \leq m} a_{u}(t, s)\right)^{2} d t d s \\
& \rightarrow\|C(t, s)\|^{2}
\end{aligned}
$$

as $m \rightarrow \infty$. Hence we obtain that for $n$ and $m$ sufficiently large

$$
\begin{aligned}
\mid \iint \frac{1}{h} \sum_{|u|,|v| \leq m} \sum_{r=b_{1}(u, v, n)}^{b_{2}(u, v, n)} K^{2}\left(\frac{r}{h}\right) a_{u}(t, s) a_{v}(t, s) d t d s \\
-\|C(t, s)\|^{2} \int_{-c}^{c} K^{2}(t) d t \mid<\varepsilon / 4 .
\end{aligned}
$$

Therefore by combining (5.1)-(5.6) it follows that

$$
\lim _{n \rightarrow \infty} \iint q_{2, n}(t, s) d t d s=\|C(t, s)\|^{2} \int_{-c}^{c} K^{2}(t) d t
$$


Since

$$
\iint \sum_{|u|,|v| \leq m} a_{u}(t, t) a_{v}(s, s) d t d s=\left(\int \sum_{|u| \leq m} a_{u}(t, t) d t\right)^{2} \rightarrow\left(\int C(t, t) d t\right)^{2},
$$

as $m \rightarrow \infty$, a small modification of the argument used to establish (5.7) yields that

$$
\lim _{n \rightarrow \infty} \iint q_{3, n}(t, s) d t d s=\left(\int C(t, t) d t\right)^{2} \int_{-c}^{c} K^{2}(t) d t .
$$

Finally

$$
\begin{aligned}
& \left|\iint q_{1, n}(t, s) d t d s\right| \\
& \quad \leq \frac{1}{h} \sup _{-c \leq x \leq c} K^{2}(x) \sum_{g, \ell=-h}^{h} \sum_{r=-(n-1)}^{n-1}\left|\iint \Psi_{\ell, r, r+g}(t, s) d t d s\right| \rightarrow 0
\end{aligned}
$$

as $n \rightarrow \infty$ by (2.3). The proposition then follows from (5.7)-(5.9).

\subsection{Proof of Theorem 2.2}

To simplify the notation below let $c_{j}=\left(E\left\|X_{0}-X_{0, j}\right\|^{4}\right)^{1 / 4}$. Then

$$
c_{j}=O\left(j^{-\alpha}\right)
$$

by assumption. Clearly

$$
\begin{aligned}
& \sum_{g, \ell=-h}^{h} \sum_{r=-(n-1)}^{n-1}\left|\iint \Psi_{\ell, r, r+g}(t, s) d t d s\right| \\
&=\sum_{\ell=0}^{h} \sum_{g=0}^{h} \sum_{r=0}^{n-1}\left|\iint \Psi_{\ell, r, r+g}(t, s) d t d s\right| \\
&+\sum_{\ell=0}^{h} \sum_{g=0}^{h} \sum_{r=-(n-1)}^{-1}\left|\iint \Psi_{\ell, r, r+g}(t, s) d t d s\right|+\ldots
\end{aligned}
$$




$$
+\sum_{\ell=-h}^{-1} \sum_{g=-h}^{-1} \sum_{r=-(n-1)}^{-1}\left|\iint \Psi_{\ell, r, r+g}(t, s) d t d s\right|
$$

where the right hand side contains eight terms corresponding to the combinations of the indices $\ell, g$, and $r$ being allowed to take either nonnegative or negative values. First we calculate a bound for the first term on the right hand side of (5.11). Let $R_{1}=\{(\ell, g, r): \ell \leq r \leq r+g, 0 \leq \ell, g \leq h, 0 \leq$ $r \leq n-1\}, R_{2}=\{(\ell, g, r): r \leq \ell \leq r+g, 0 \leq \ell, g \leq h, 0 \leq r \leq n-1\}$, and $R_{3}=\{(\ell, g, r): r \leq r+g \leq \ell, 0 \leq \ell, g \leq h, 0 \leq r \leq n-1\}$. Then

$$
\begin{array}{r}
\sum_{\ell=0}^{h} \sum_{g=0}^{h} \sum_{r=0}^{n-1}\left|\iint \Psi_{\ell, r, r+g}(t, s) d t d s\right| \leq \sum_{R_{1}}\left|\iint \Psi_{\ell, r, r+g}(t, s) d t d s\right| \\
+\sum_{R_{2}}\left|\iint \Psi_{\ell, r, r+g}(t, s) d t d s\right|+\sum_{R_{3}}\left|\iint \Psi_{\ell, r, r+g}(t, s) d t d s\right| .
\end{array}
$$

By the definition of the $\Psi_{\ell, r, r+g}(t, s)$ and the triangle inequality it follows that

$$
\begin{aligned}
\frac{1}{h} \sum_{R_{1}}\left|\iint \Psi_{\ell, r, r+g}(t, s) d t d s\right| \\
\leq \leq \frac{1}{h} \sum_{R_{1}}\left\{\left|\iint a_{r}(t, t) a_{r+g-\ell}(s, s) d t d s\right|+\left|\iint a_{r-\ell}(t, s) a_{r+g}(t, s) d t d s\right|\right\} \\
\quad+\frac{1}{h} \sum_{R_{1}}\left|\iint E X_{0}(t) X_{\ell}(s) X_{r}(t) X_{r+g}(s)-a_{\ell}(t, s) a_{g}(t, s) d t d s\right|
\end{aligned}
$$

By the inequality $\left|E \int X_{0}(t) X_{j}(t) d t\right| \leq\left(E\left\|X_{0}\right\|^{2}\right)^{1 / 2}\left(E\left\|X_{0}-X_{0, j}\right\|^{2}\right)^{1 / 2}$ (cf. (A.1) in [12]) and the fact that $\left(E \zeta^{2}\right)^{1 / 2} \leq\left(E \zeta^{4}\right)^{1 / 4}$, we have that

$$
\left|\iint a_{r}(t, t) a_{r+g-\ell}(s, s) d t d s\right| \leq E\left\|X_{0}\right\|^{2} c_{r} c_{r+g-\ell}
$$


and

$$
\left|\iint a_{r-\ell}(t, s) a_{r+g}(t, s) d t d s\right| \leq E\left\|X_{0}\right\|^{2} c_{r-\ell} c_{r+g} .
$$

It follows that

$$
\begin{aligned}
\sum_{R_{1}}\left|\iint a_{r}(t, t) a_{r+g-\ell}(s, s) d t d s\right| & \leq E\left\|X_{0}\right\|^{2} \sum_{R_{1}} c_{r} c_{r+g-\ell} \\
& =E\left\|X_{0}\right\|^{2} \sum_{\ell=0}^{h} \sum_{r=\ell}^{n-1} c_{r} \sum_{g=\ell-r}^{h} c_{r+g-\ell} \\
& \leq E\left\|X_{0}\right\|^{2}\left(\sum_{\ell=0}^{\infty} \sum_{r=\ell}^{\infty} c_{r}\right)\left(\sum_{g=0}^{\infty} c_{g}\right)<\infty
\end{aligned}
$$

using (5.10). Similarly

$$
\begin{aligned}
\sum_{R_{1}}\left|\iint a_{r-\ell}(t, s) a_{r+g}(t, s) d t d s\right| & \leq E\left\|X_{0}\right\|^{2} \sum_{R_{1}} c_{r-\ell} c_{r+g} \\
& =E\left\|X_{0}\right\|^{2} \sum_{\ell=0}^{h} \sum_{r=\ell}^{n-1} \sum_{g=\ell-r}^{h} c_{r-\ell} c_{r+g} \\
& =E\left\|X_{0}\right\|^{2} \sum_{\ell=0}^{h} \sum_{r=\ell}^{n-1} \sum_{p=\ell}^{h-r} c_{r-\ell} c_{p} \\
& \leq E\left\|X_{0}\right\|^{2}\left(\sum_{\ell=0}^{\infty} \sum_{p=\ell}^{\infty} c_{p}\right)\left(\sum_{r=0}^{\infty} c_{r}\right)<\infty
\end{aligned}
$$

Therefore

$$
\begin{aligned}
\lim _{n \rightarrow \infty} \frac{1}{h} \sum_{R_{1}}\left\{\left|\iint a_{r}(t, t) a_{r+g-\ell}(s, s) d t d s\right|\right. \\
\left.+\left|\iint a_{r-\ell}(t, s) a_{r+g}(t, s) d t d s\right|\right\}=0
\end{aligned}
$$


since $h \rightarrow \infty$ as $n \rightarrow \infty$. With $\alpha$ defined by (5.10), let $\xi(n)=h^{\kappa}$ where $\kappa=$ $(\alpha+2) /(6(\alpha-1))$. Due to the fact that $\alpha>4$ it follows that $\xi(n)^{1-\alpha} h \rightarrow 0$ and $\xi^{3}(n) / h \rightarrow 0$ as $n \rightarrow \infty$. Let $R_{1,1}=\left\{(\ell, g, r) \in R_{1}: r-\ell>\xi(n)\right\}$, and $R_{1,2}=\left\{(\ell, g, r) \in R_{1}: r-\ell \leq \xi(n)\right\}$ so that $R_{1}=R_{1,1} \cup R_{1,2}$. It follows from the inequality

$$
\operatorname{Cov}\left(\int X_{0}(t) X_{j}(t) d t, \int X_{k}(s) X_{\ell}(s) d s\right) \leq\left(E\left\|X_{0}\right\|^{4}\right)^{3 / 4}\left(c_{k-j}+c_{\ell-j}\right),
$$

shown as (A.9) in [12], that there exists a constant $A_{1}$ depending only on the distribution of $X_{0}$ such that for all $(\ell, g, r) \in R_{1,1}$

$$
\left|\iint E X_{0}(t) X_{\ell}(s) X_{r}(t) X_{r+g}(s)-a_{\ell}(t, s) a_{g}(t, s) d t d s\right| \leq A_{1} c_{r-\ell} .
$$

Thus by (5.10) we obtain that

$$
\begin{aligned}
\sum_{R_{1,1}} \frac{1}{h} \mid & \iint E X_{0}(t) X_{\ell}(s) X_{r}(t) X_{r+g}(s)-a_{\ell}(t, s) a_{g}(t, s) d t d s \mid \\
& \leq \frac{A_{1}}{h} \sum_{\ell=0}^{h} \sum_{r=\ell+\xi(n)}^{n} \sum_{g=\ell-r}^{h} c_{r-\ell} \\
& \leq A_{1} h \sum_{p=\xi(n)}^{\infty} c_{p}=O\left(h \xi(n)^{1-\alpha}\right) \rightarrow 0
\end{aligned}
$$

as $n \rightarrow \infty$. To obtain a bound over $R_{1,2}$ we write $R_{1,2}=\cup_{i=1}^{3} R_{1,2, i}$ where $R_{1,2,1}=\left\{(\ell, g, r) \in R_{1,2}: \ell>\xi(n)\right\}, R_{1,2,2}=\left\{(\ell, g, r) \in R_{1,2}: g>\xi(n)\right\}$, $R_{1,2,3}=\left\{(\ell, g, r) \in R_{1,2}: \ell, g \leq \xi(n)\right\}$. It follows since

$$
\left|E \iint X_{0}(t) X_{j}(t) X_{k}(s) X_{\ell}(s) d t d s\right| \leq 3\left(E\left\|X_{0}\right\|^{4}\right)^{3 / 4} c_{j}
$$

(cf. (A.4) in [12]) that for $(\ell, g, r) \in R_{1}$ with some constant $A_{2}$ 


$$
\left|\iint E X_{0}(t) X_{\ell}(s) X_{r}(t) X_{r+g}(s) d t d s\right| \leq A_{2} \min \left\{c_{\ell}, c_{g}\right\} .
$$

Therefore by again using (5.10)

$$
\begin{aligned}
& \frac{1}{h} \sum_{R_{1,2,1}} \mid \iint E X_{0}(t) X_{\ell}(s) X_{r}(t) X_{r+g}(s) d t d s \mid \\
& \leq \frac{A_{2}}{h} \sum_{\xi(n)+1}^{h} \sum_{r=\ell}^{\ell+\xi(n)} \sum_{g=\ell-r}^{h} c_{\ell} \\
& \leq A_{2} \xi(n) \sum_{\ell=\xi(n)}^{\infty} c_{\ell}=O\left(\xi(n)^{2-\alpha}\right) \rightarrow 0
\end{aligned}
$$

as $n \rightarrow \infty$. Similarly $(1 / h) \sum_{R_{1,2,2}}\left|\iint E X_{0}(t) X_{\ell}(s) X_{r}(t) X_{r+g}(s) d t d s\right| \rightarrow 0$. A simple calculation gives that, $(1 / h) \sum_{R_{1,2,3}}\left|\iint E X_{0}(t) X_{\ell}(s) X_{r}(t) X_{r+g}(s) d t d s\right|=$ $O\left(\xi(n)^{3} / h\right) \rightarrow 0$ which shows that

$$
\frac{1}{h} \sum_{R_{1,2}}\left|\iint E X_{0}(t) X_{\ell}(s) X_{r}(t) X_{r+g}(s) d t d s\right| \rightarrow 0
$$

Furthermore we obtain by using the definition of the sets $R_{1,2, j}$ and (5.10) that

$$
\begin{aligned}
\frac{1}{h} \sum_{R_{1,2}}\left|\iint a_{\ell}(t, s) a_{g}(t, s) d t d s\right| & \leq \sum_{i=1}^{3} \sum_{R_{1,2, i}}\left|\iint a_{\ell}(t, s) a_{g}(t, s) d t d s\right| \\
& =O\left(\xi(n)^{2-\alpha}\right)+O\left(\xi^{3}(n) / h\right) \rightarrow 0 .
\end{aligned}
$$

Combining (5.14)-(5.16) gives that

$$
\frac{1}{h} \sum_{R_{1}}\left|\iint E X_{0}(t) X_{\ell}(s) X_{r}(t) X_{r+g}(s)-a_{\ell}(t, s) a_{g}(t, s) d t d s\right| \rightarrow 0 .
$$


This result combined with (5.13) and (5.12) shows that

$$
\frac{1}{h} \sum_{R_{1}}\left|\iint \Psi_{\ell, r, r+g}(t, s) d t d s\right| \rightarrow 0 .
$$

Similar arguments show that the sums over $R_{2}$ and $R_{3}$ also tend to zero and hence

$$
\frac{1}{h} \sum_{\ell=0}^{h} \sum_{g=0}^{h} \sum_{r=0}^{n-1}\left|\iint \Psi_{\ell, r, r+g}(t, s) d t d s\right| \rightarrow 0
$$

as $n \rightarrow \infty$. Since the process $\left\{X_{i}(t),-\infty<i<\infty, t \in[0,1]\right\}$ is assumed to be strictly stationary we get that

$$
\sum_{\ell=0}^{h} \sum_{g=0}^{h} \sum_{r=-(n-1)}^{-1}\left|\iint \Psi_{\ell, r, r+g}(t, s) d t d s\right|=\sum_{\ell=0}^{h} \sum_{g=0}^{h} \sum_{r=1}^{n-1}\left|\iint \Psi_{\ell+r, r, g}(t, s) d t d s\right|,
$$

and hence the the arguments above show that the second term on the right hand side of (5.11) tends to zero as well. The other six terms can be handled in the same way and thus the lemma follows from (5.17).

\subsection{Proof of Proposition 2.1}

It is easy to see that

$$
E \hat{\gamma}_{i}(t, s)=\frac{n-|i|}{n} c_{i}(t, s)+r_{n, i}(t, s) \quad \text { with } \quad\left\|r_{n, i}\right\| \leq \frac{2}{n} \sum_{\ell=0}^{\infty}\left\|a_{\ell}\right\| .
$$

Thus by the triangle inequality

$$
\left\|E \hat{C}_{n, h}-\sum_{i=-(n-1)}^{n-1} K_{f}\left(\frac{i}{h}\right) c_{i}\right\|=O\left(\frac{h}{n} \sum_{\ell=1}^{\infty} \ell\left\|a_{\ell}\right\|\right) .
$$


Also, using the definition of $K_{f}(t)=K_{f}(t ; x)$ we get

$$
\left\|\sum_{i=-(n-1)}^{n-1} K_{f}\left(\frac{i}{h}\right) c_{i}-C\right\|=O\left(\sum_{\ell=\lfloor n x\rfloor+1}\left\|a_{\ell}\right\|+\frac{h}{n} \sum_{\ell=1}^{\infty} \ell\left\|a_{\ell}\right\|\right) .
$$

\subsection{Proof of Proposition 3.1}

It follows from the central limit theorem for finite dependent stationary sequences in Hilbert spaces in [11], p. 297 (cf. also [4]) that if $\left\{X_{i}(t),-\infty<\right.$ $i<\infty, t \in[0,1]\}$ is $m$-dependent then $\max _{m+1 \leq i \leq m+r}\left\|\hat{\gamma}_{i}\right\|=O_{p}(1 / \sqrt{n})$ for any $r \geq 1$. Notice that $\hat{m}>m$ only if

$$
\max _{1 \leq k \leq H} \hat{\rho}_{m+k} \geq T \sqrt{\log n / n}
$$

Therefore,

$$
\begin{aligned}
P(\hat{m}>m) & \leq P\left(\max _{1 \leq k \leq H} \hat{\rho}_{m+k} \geq T \sqrt{\log n / n}\right) \\
& =P\left(O_{P}(1 / \sqrt{n}) \geq T \sqrt{\log n / n}\right) \rightarrow 0
\end{aligned}
$$

as $n \rightarrow \infty$. Now suppose $j<m$. Then for $\hat{m}=j$ at least $\hat{\rho}_{j+1}<T \sqrt{\log n / n}$. Since $\left\|\operatorname{Cov}\left(X_{0}(t), X_{i}(s)\right)\right\|>0$ for $0 \leq i \leq m$, the ergodic theorem in Hilbert space implies that $\hat{\rho}_{j+1} \stackrel{P}{\rightarrow} B>0$ as $n \rightarrow \infty$. Therefore $P(\hat{m}=j) \leq$ $P\left(\hat{\rho}_{j+1}<T \sqrt{\log n / n}\right) \rightarrow 0$. Since $j<m$ was arbitrarily chosen this implies $P(\hat{m}<m) \rightarrow 0$. Combining these results gives that $P(\hat{m}=m) \rightarrow 1$ as $n \rightarrow \infty$ which implies the proposition.

\subsection{Proof of (3.1)}

By [11], p. 297, (cf. also [4]) we obtain that if $\left\{X_{i}(t),-\infty<i<\infty, t \in\right.$ $[0,1]\}$ is an $m$-dependent sequence then for $j>m, \sqrt{n} \hat{\gamma}_{j}(t, s) \stackrel{D[0,1]^{2}}{\rightarrow} \Gamma_{j}(t, s)$, where $\Gamma_{j}(t, s)$ is a Gaussian process with mean $E \Gamma_{j}(t, s)=0$ and nonnegative definite covariance function $E \Gamma_{j}(t, s) \Gamma_{j}\left(t^{\prime}, s^{\prime}\right)=C_{j}\left(t, t^{\prime}, s, s^{\prime}\right)$. Then by Mercer's Theorem there exist non-negative eigenvalues $\lambda_{i, j}, 1 \leq i<\infty$ and a corresponding collection of orthonormal eigenfunctions $\phi_{i, j}(t, s), 1 \leq$ 
$i<\infty, 0 \leq t, s, \leq 1$ so that

$$
\iint C_{j}\left(t, t^{\prime}, s, s^{\prime}\right) \phi_{i, j}\left(s, s^{\prime}\right) d s d s^{\prime}=\lambda_{i, j} \phi_{i, j}\left(t, t^{\prime}\right) .
$$

Hence, by the Karhunen-Loéve expansion, $\Gamma_{j}(t, s)=\sum_{\ell=1}^{\infty} \lambda_{\ell, j}^{1 / 2} N_{\ell, j} \phi_{\ell, j}(t, s)$, where $\left\{N_{\ell, j}\right\}_{\ell=1}^{\infty}$ are iid standard normal random variables. Therefore

$$
\sqrt{n}\left\|\hat{\gamma}_{j}\right\| \stackrel{D}{\rightarrow}\left(\sum_{\ell=1}^{\infty} \lambda_{\ell, j} N_{\ell, j}^{2}\right)^{1 / 2} .
$$

Finally it follows by the ergodic theorem in Hilbert spaces that

$$
\sqrt{n} \hat{\rho}_{j} \stackrel{D}{\rightarrow}\left(\sum_{\ell=1}^{\infty} \lambda_{\ell, j} N_{\ell, j}^{2}\right)^{1 / 2} / \int E X_{0}^{2}(t) d t .
$$

\section{References}

[1] Andrews, D. W. K., 1991. Heteroskedasticity and autocorrelation consistent covariance matrix estimation. Econometrica 59, 817-858.

[2] Andrews, D. W. K., Monahan, J. C., 1992. An improved heteroskedasticity and autocorrelation consistent covariance matrix estimator. Econometrica 60, 953-966.

[3] Beltrao, K., Bloomfield, P., 1987. Determining the bandwidth of a kernel spectrum estimate. Journal of Time Series Analysis 8, 21-38.

[4] Bosq, D., 2000. Linear Processes in Function Spaces. Springer, New York.

[5] Bühlmann, P., 1996. Locally adaptive lag-window spectral estimation. Journal of Time Series Analysis 17, 247-270.

[6] Ferraty, F., Vieu, P., 2006. Nonparametric Functional Data Analysis: Theory and Practice. Springer. 
[7] Hörmann, S., Horváth, L., Reeder, R., 2013. A functional version of the ARCH model. Econometric Theory 29, 267-288.

[8] Hörmann, S., Kokoszka, P., 2012. Functional time series. In: Rao, C. R., Rao, T. S. (Eds.), Time Series. Vol. 30 of Handbook of Statistics. Elsevier.

[9] Horváth, L., Kokoszka, P., Reeder, R., 2012. Estimation of the mean of functional time series and a two sample problem. Journal of the Royal Statistical Society (B) 74, 103-122.

[10] Horváth, L., Kokoszka, P., Rice, G., 2014. Testing stationarity of functional time series. Journal of Econometrics 179, 66-82.

[11] Horváth, L., Kokoszka, P. S., 2012. Inference for Functional Data with Applications, 1st Edition. Springer.

[12] Horváth, L., Rice, G., 2014. Testing independence between functional time series. Journal of Econometrics 00, forthcoming, http://arxiv.org/abs/1403.5710.

[13] Jirák, M., 2013. On weak invariance principles for sums of dependent random functionals. Statistics and Probability Letters 83, 2291-2296.

[14] Kokoszka, P. S., Miao, H., Zhang, X., 2014. Functional dynamic factor model for intraday price curves. Journal of Financial Econometrics 0, $1-22$.

[15] Liu, W., Wu, W. B., 2010. Asymptotics of spectral density estimates. Econometric Theory 26, 1218-1245.

[16] Politis, D. N., 2003. Adaptive bandwidth choice. Journal of Nonparametric Statistics 25, 517-533.

[17] Politis, D. N., Romano, J. P., 1996. On flat-top spectral density estimators for homogeneous random fields. Journal of Statistical Planning and Inference 51, 41-53.

[18] Politis, D. N., Romano, J. P., 1999. Multivariate density estimation with general flat-top kernels of infinite order. Journal of Multivariate Analysis $68,1-25$. 
[19] Priestly, M. B., 1981. Spectral Analysis and Time Series. Academic Press.

[20] Rosenblatt, M., 1991. Stochastic Curve Estimation, 1st Edition. Institute of Mathematical Statistics. 